\title{
Why the Evolution of Heritable Symbiosis Neither Enhances Nor Diminishes the Fitness of a Symbiont
}

\author{
Adrian Stencel*
}

\begin{abstract}
One of the current problems in microbiology concerns the understanding of fitness in hostsymbiont systems. A great deal of research and conceptual work has analysed how the host benefits from such associations; however, very little of this work has attempted to take the microbial perspective. Nevertheless, some scientists have argued that we should conduct more comparative studies of both microorganisms that interact with a host and their freeliving counterparts in order to determine whether or not symbiosis is beneficial for these microorganisms. In this paper, by means of analysing heritable symbiosis as a case study, I intend to provide a different perspective on this subject. Mainly, I argue that asking how the fitness of a given microorganism was changed during the evolution of heritable symbiosis may be theoretically unjustified at both the early and late stages of this process. The reason for this, I argue, is that, during the evolution of heritable symbiosis, the fitness of a microorganism becomes incommensurable with that of its free-living counterparts because their contexts become radically different; therefore, comparing their fitness makes little sense. My analysis is driven by the use of the ecological concept of fitness; accordingly, my conclusions are bound to this specific interpretation of fitness.
\end{abstract}

$$
\begin{gathered}
\text { Keywords } \\
\text { symbiosis } \bullet \text { heritability } \bullet \text { symbiogenesis } \bullet \text { niche construction }
\end{gathered}
$$

Part of a special issue, Contributed Papers from the Interdisciplinary Workshop on Holobionts, guest-edited by Derek Skillings. ${ }^{\dagger}$

\section{Introduction}

Host-microbe interactions have been studied mainly from the perspective of the host; thus, many studies have attempted to determine how these interactions influence the fitness of hosts

*Institute of Philosophy, Jagiellonian University, Poland, adstencel@gmail.com

†The Interdisciplinary Workshop on Holobionts was held in Bordeaux, November 6-8, 2017, and received funding from the European Research Council (ERC) under the European Union's Horizon 2020 Research and Innovation Programme, grant agreement \#637647.

Received 16 September 2018; Revised 22 January 2020; Accepted 26 May 2020 doi:10.3998/ptpbio.2098

๑ OPEN ACCESS - PTPBIO.ORG 
(e.g., Kikuchi et al. 2007; Skillings 2016; Suárez 2018; Stencel and Wloch-Salamon 2018). However, it has been noted recently that we don't know how these kinds of interactions influence the fitness of microbes; hence the need to compare the fitness of microbes that interact with a host with their free-living counterparts, in order to determine whether participation in these interactions offers any fitness benefits for the former (Garcia and Gerardo 2014; Mushegian and Ebert 2016; Lowe et al. 2016). In the present paper I attempt to provide a different perspective on this problem. Mainly, I argue that sometimes it may be impossible to understand how host-microbe interactions shape the fitness of microbes, because it has become incommensurable with that of their free-living counterparts (Stencel 2016). I demonstrate that this happens in the case of heritable symbionts, i.e., symbiotic microorganisms that are transmitted vertically from one generation of hosts to another. The best-known examples of heritable symbiosis include cellular organelles, such as mitochondria and chloroplasts, and the symbiotic bacterium Buchnera (Moran et al. 2008).

Of course, before one can say that the fitness of certain units is incommensurable, one must specify what is meant by fitness, as there is a great deal of ambiguity concerning this concept, which, over the past few decades, has been the subject of many debates (Matthen and Ariew 2002; Ariew and Lewontin 2004; Godfrey-Smith 2014; Rosenberg and Bouchard 2015; Triviño and Nuño de la Rosa 2016). Here, I prefer to think about the fitness of heritable symbionts from the point of view of evolution, as this problem frequently appears in the field of evolutionary biology. Specifically, scientists, unsure of whether becoming a heritable symbiont enhances or diminishes the fitness of microbes, use metaphors such as 'slavery' (Maynard Smith and Szathmáry 1995, 141; Dyall et al. 2004). In spite of this, I discuss the aforementioned problems in the context of the ecological concept of fitness (Matthen and Ariew 2002; Ariew and Lewontin 2004; Rosenberg and Bouchard 2015), because this concept captures the 'essence' of Darwinism by emphasising that fitness is a certain relationship between organisms and their environment (Ariew and Lewontin 2004). Thus, the conclusions of my analysis are bound to this specific interpretation of fitness.

The structure of the paper is as follows. In the next section I present the concept of ecological fitness and show when, within the context of this concept, the fitness of certain units is commensurable. Then I argue that the ecological fitness of heritable symbionts (at both the early and late stages of co-evolution with their hosts) is incommensurable with that of their freeliving counterparts because the necessary conditions for comparison have not been fulfilled, and therefore we are not in a position to argue that the ecological fitness of the microorganisms in question is enhanced or diminished during the evolution of heritable symbiosis. I conclude the paper with some general remarks aimed at linking my analysis to the idea of niche construction (Odling-Smee and Laland 2011; Laland et al. 2016).

\section{Ecological Fitness and Its Commensurability}

The ecological concept sees fitness as a kind of relationship between an organism and its environment (Matthen and Ariew 2002; Rosenberg and Bouchard 2015). Organisms are not isolated objects; rather, they inhabit environments composed of other organisms and inorganic factors. Therefore, in order to survive and reproduce, organisms must deal with the obstacles that the environment 'designs' for them. This may require dealing with certain pathogens, finding a sexual mate, surviving within a certain range of temperatures, catching prey, etc. Thus, if one organism is supposed to be fitter, in ecological terms, than another, it must be capable of solving these problems more efficiently. Thus, following Rosenberg and Bouchard (2015), we might 
define ecological fitness as follows: ' $x$ is fitter than $y$ if and only if $x$ 's traits enable it to solve the "design problems" set by the environment more fully than $y$ 's traits do'.

As simple as this concept sounds, it is not easy to evaluate which organism is characterised by a higher level of ecological fitness, because it is not clear how to distinguish these design problems, i.e., how to separate one such problem from another (Rosenberg and Bouchard 2015); thus, it would be very problematic to directly compare the ability to solve these problems. As a result, we need to figure out how to do this indirectly by using proxies- to use Bourrat's (2015) vocabulary - of some kind which would inform us more or less precisely about differences in ecological fitness. The most popular way of measuring fitness is to focus on reproductive success (Godfrey-Smith 2009); thus this method may be a good candidate, and I'm going to stick with it. However, comparing the reproductive success of two individuals is not equivalent to saying that one is characterised by a higher level of ecological fitness. Before using reproductive output as a proxy for ecological fitness, certain conditions must be fulfilled. Let me say a few words about them.

The first very important condition concerns the environment. In order to make reproductive success serve as a proxy for ecological fitness, the organisms in question must be placed in the same environment. The above paragraphs provide some clues as to why this is so. Mainly, from the perspective of the ecological concept of fitness, it makes little sense to compare the fitness of units inhabiting different environments. In such a situation, our measurements of reproductive success would not mirror a superior ability to solve design problems, for the simple reason that, when organisms are placed in different environments, they are exposed to different factors (Bourrat 2015; Stencel 2016). To survive and reproduce, they need to solve the different sets of problems found in their respective environments. This can be easily understood by considering an extreme example. Suppose we have two cats-genetic clones-one living in a city and the other on the moon. The former leaves ten offspring, the latter none. Does this mean that the latter is less fit in ecological terms than the former? No, not really. The latter leaves no offspring because it's exposed on the moon to totally different factors (lack of food, different atmosphere, the absence of individuals of the opposite sex necessary for reproduction, etc.). Therefore, the difference in reproductive output here derives not from the possession by one cat of superior traits that enable it to solve a given set of problems more effectively (which is impossible, since they are clones!), but from the fact of each cat being confronted by different environmental obstacles. This shows that if we want to make reproductive success serve as a proxy for ecological fitness, reproduction has to be measured in the same environment.

Unfortunately, the condition of inhabiting the same environment is not sufficient to ensure that two organisms have to solve the same obstacles in order to reproduce and survive. Whether a given factor constitutes an obstacle for a given organism depends on the traits the organism possesses. Indeed, it depends on its phenotype, which comprises 'the physical and behavioural traits of the organism, for example, size and shape, metabolic activities, and patterns of movement' (Taylor and Lewontin 2017). Thus, if we wish to compare the ecological fitness of certain units, we should limit our investigations to organisms with similar phenotypes. In other words, they must possess the same traits. Why is this similarity necessary? Because a phenotype, defined as all the traits of an organism, is what interacts with the environment. Thus, the phenotype of a given organism is confronted with the design problems generated by a given structure of the environment. However, phenotypes differ between one species and another; thus different phenotypes might experience a given environment in different ways, as they differ in many basic traits. The relevant organisms may live (physically) in the same place, but because of differences in their phenotypes, they have to overcome different obstacles. For example, the presence of a given pathogen may be a problem that a chimpanzee-but not necessarily a cat-must 'solve' if 
it wants to survive, as this pathogen may have the ability to infect chimps only. Thus it seems that if we want to make justifiable comparisons of fitness, we need to compare the reproductive success of units with similar phenotypes, as this makes it much more probable that, when placed in the same environment, they will be exposed to the same obstacles.

Accordingly, it seems that it makes sense to use reproductive success as a proxy for the ecological fitness of certain units only when those units are (i) phenotypically similar and (ii) placed in the same environment. In most cases there are no problems associated with comparing the ecological fitness of individuals. For example, students of experimental evolution very often study the evolution of members of a given species to determine how they deal with certain external factors. As Kawecki et al. $(2012,547)$ concluded: 'Many evolution experiments seek to understand how populations adapt to particular environmental conditions, usually defined in terms of a particular factor, such as temperature, nutrition, other environmental stressors, parasites, or competition'. Presumably, comparisons of ecological fitness in such situations would not lead to any significant disagreements, as they involve very similar individuals exposed in the laboratory to the same environmental forces. Thus, in such cases, if one unit fares better than another, it is quite obvious to suggest that it is characterised by a higher level of fitness (in ecological terms), because it does so while struggling with the same environmental factors. Unfortunately, the process of evolution in nature is unlike the same process in experimental settings, because the conditions necessary to make reliable comparisons are not always in force. In the next section I will show that this is true in the case of heritable symbiosis.

\section{The Fitness of Heritable Symbionts}

Heritable symbionts are symbiotic microorganisms transmitted vertically from one generation of hosts to another. One example of this kind of symbiosis is the eukaryotic cell, in which a host engulfs a free-living bacterium and subsequently (on an evolutionary time scale) transforms it into a mitochondrion, which is then transmitted vertically along with many other elements when the cell reproduces (Maynard Smith and Szathmáry 1995; Zimorski et al. 2014). To cite another example, the symbiotic bacteria Buchnera sp. provide necessary nutrients for aphids and are transmitted from generation to generation via special propagules (Moran et al. 2008).

The existence of heritable symbiosis raises the question of whether it enhances or diminishes the fitness of the endosymbiont in comparison to that of its free-living counterparts. In other words, if an individual from a given environment becomes a heritable symbiont, is it superior or inferior, in terms of fitness, to similar individuals from the same environment? This question has been a source of great puzzlement. How the fitness of a symbiont changes during the evolution of heritable symbiosis is not very clear. Instances of this process (especially the endosymbiotic origin of mitochondria) are ancient events, and thus we have no way of comparing the fitness of heritable symbionts against that of their free-living counterparts to see whether it was enhanced or diminished. This concern is expressed most clearly by Maynard Smith and Szathmáry (1995):

Although endosymbionts are often regarded as mutualistic, it may be very difficult to test this assertion. It is easy to demonstrate that the association is beneficial to the host, but impossible to prove the opposite, since free-living organelles are absent, and thus the increase in survival, growth rate and fecundity is impossible to measure. To say that organelles benefit from the association because they die without the host cell is to confuse obligate dependence with benefit. Thus, we are inclined to think that endosymbiotic organelles are encapsulated slaves. (141) 
Therefore, it seems the main problem here is a lack of data. Because most events leading to the evolution of heritable symbiosis are now ancient, heritable symbionts have no free-living counterparts; therefore, we cannot compare their fitness with that of their free-living counterparts to determine whether the process enhanced or diminished it. Thus, we are doomed to use more or less useful metaphors, such as slavery (Maynard Smith and Szathmáry 1995, 141; Dyall et al. 2004). This approach, however, presupposes that such a comparison of fitness is possible, at least in theory. But is this really the case? Is the fitness of the heritable symbiont comparable with its free-living counterparts, even theoretically? Here I would argue that their fitness is incommensurable at both early and late stages of the evolution of heritable symbiosis. Let's start with the former.

Suppose that we have a population of microorganisms that inhabit a certain environment and are of essentially the same phenotype, which assures that they are exposed to the same factors in this environment. Let's say that they live in a certain part of the ocean, and let's assume as well that there are certain obstacles that these microorganisms have to overcome, such as pathogens, predators, limited access to nutrients, and certain toxins, if they are to survive and reproduce. Indeed, in this environment these organisms are confronted with a certain set of design problems. Those microorganisms that deal with those obstacles more effectively (for example, through immunity to pathogens, or the ability to degrade toxins) will end up with a greater number of offspring. However, suppose that one of the microorganisms, after being engulfed, but not digested, by a predator, begins instead to live inside it. As a result, let's assume that, as a result of living inside the host, the microbe leaves twice as many offspring as the most successful microbe inhabiting the environment outside the host. In the context of the ecological concept of fitness, does this greater rate of reproduction make the microbe living inside the host fitter than those living outside?

Let's ask this question in a different way. Are the conditions assumed in the last section (similarity of phenotype and environment) fulfilled in this situation, so that we can draw conclusions about superiority or inferiority in terms of ecological fitness? Not really. What happens in this situation is that an organism's primary environment is changed drastically when it begins life in a new habitat, one that is totally different in many aspects. Whereas outside the host it had to degrade some toxins, maintain its immunological system in order to defend itself against pathogens, etc., when it begins life inside the host it no longer has to contend with these environmental obstacles. Indeed, inside the host it may no longer need to defend itself against pathogens or to degrade toxins at all, since the host may not allow them to enter. The engulfed microbe may even obtain access to more resources. As result it doesn't need to invest so much energy in those processes and thus can reproduce at a higher rate. Of course, the environment inside the host, because of its biochemical content, may influence the microbe such that its rate of reproduction declines. In fact, it doesn't really matter what the result would be. The point is that the first step of the evolution of heritable symbiosis (engulfment) is huge, changing the microbe's environment completely. As a result, inside the host the microbe is confronted with different environmental problems; thus, whether its reproduction rate rises or falls, it makes no sense to argue that the level of its ecological fitness is higher or lower than that of free-living microbes, simply because it now faces different design problems. In other words, the second condition necessary for reliable comparisons of ecological fitness has not been fulfilled.

What about the later stages of the evolution of heritable symbiosis? Does the situation change in any way? I think that, in terms of fitness commensurability, it gets even worse. What follows is the evolution of this microbe in a new environment (inside the host), which leads to evolution of the phenotype of the heritable symbiont in a different direction than that of its freeliving counterparts. There are two main reasons why this happens. Firstly, as the environment 
inside the host is very different than the world outside, different genes will be under pressure; therefore, they may evolve to 'fit' the demands of the environment. Furthermore, many genes might lose their importance, which is easy to understand, as the threats inside the host are quite different than the threats outside the host. As a result, many genes may be lost. I don't need to speculate at length, since, when we look at heritable symbionts right now (such as Buchnera in aphids or mitochondria in an eukaryotic cell), we can readily observe how much they differ, in many aspects, from free-living bacteria. Their phenotype is completely distinct. This is accompanied by extreme changes in their genome; they've lost many genes, whereas others have been transferred to the host's genome, and such genes as they've kept have undergone sequence evolution (Moran et al. 2008; Suárez 2016). Secondly, there is not much room for restoring the genes that have been lost or for the transfer of novel genes, since gene flow between freeliving microbes and heritable symbionts, which is considered to be one of the most important factors in keeping phenotypes alike (see Templeton 1989), is limited. This is simply because heritable symbionts have very few opportunities to interact with their free-living counterparts. In other words, when a new gene that codes for a certain phenotype appears, for example, in a bacterium outside the host, it is very unlikely that it will be found within the genome of heritable symbionts. This clears the way for further diversification of the lineages of heritable symbionts and of their free-living counterparts. Taken together, comparing the reproductive rate 1 of heritable symbionts with that of either their free-living ancestors (which would now have to be 'living fossils') or their current free-living descendants would make very little sense, because they occupy different environments and their phenotypes and genotypes are different. Thus, such a comparison would be similar to comparing a cat that lives in environment $A$ and a dog that lives in environment $B$. Such comparisons may yield some information, but certainly nothing about differences in the ecological fitness of the units in question.

To sum up, in the context of the ecological concept of fitness it makes no sense to argue that becoming a heritable symbiont enhances or diminishes the fitness of a microorganism in comparison to that of its free-living counterparts. At an early stage, a microorganism is no better 'adapted', thanks to this process, to overcoming certain problems that it encounters in its prehost environment. Instead, it is confronted with different problems, because its environment, once it starts to live inside the cell, is totally different from the outside world inhabited by its free-living counterparts. As a result, its ecological fitness becomes incommensurable with that of those counterparts from the very beginning of the evolution of heritable symbiosis. What is more, at later stages of this process it makes even less sense to compare their fitness, because, through living in a different environment, heritable symbionts evolve very different phenotypes; thus making comparison of their reproductive outputs even less justified.

\section{Concluding Remarks}

In the above sections I've presented the conditions necessary for reliable comparisons of ecological fitness and argued that in the case of the evolution of heritable symbiosis they are not fulfilled. Thus, to state that becoming a heritable symbiont either enhances or diminishes the fitness of a

\footnotetext{
${ }^{1}$ One might wonder whether, at this stage of the evolution of heritable symbiosis, it makes sense to focus on the reproductive success of the symbiont, as at this point it is highly integrated into the body of the host and reproduces along with the host. Perhaps, then, we should focus on the reproductive success of the whole. In this scenario (Stencel 2016), nothing would change very much; rather, it would make the point even more vividly, because the phenotype of the whole (host + heritable symbiont) would then diverge even more widely than that of the heritable symbiont alone from the phenotypes of the free-living counterparts of the heritable symbiont. This can be easily seen if we recall that the host might be, for example, an aphid.
} 
microorganism is unjustified. Accordingly, I suggested a different approach to the problem, current in microbiology (Gerardo and Garcia 2014, Mushegian and Ebert 2016; Lowe et al. 2016), of understanding the fitness of microbes in a host-microbe symbiotic system. I chose to analyse heritable symbiosis, because understanding the fitness of these symbionts has been problematic for a long time (see e.g., Maynard Smith and Szathmáry 1995, 141; Dyall et al. 2004). This, however, is not the end of the story, since many microbes are not heritable; rather, they are acquired from an environment and live both outside and inside their hosts, so there is a need to analyse them as well. However, this topic exceeds the scope of this paper; accordingly, I plan to return to it in the near future.

Now the question is: Can we generalise based on the points I've made? In other words, can my work be related to more general principles that would explain my results? In this respect, I think the concept of niche construction (Odling-Smee and Laland 2011; Laland et al. 2016) may be a good candidate. Niche construction 'is the process whereby organisms, through their metabolism, activities, and choices, modify their own and/or each other's niches' (Odling-Smee and Laland 2011). In other words, this concept assumes that the environment of a given organism is not a fixed system into which the organism has to fit; rather, it may be modified by the actions of this organism or others. This may happen in a more conscious manner, as when human beings transform their environment by building cities, or less consciously, as when a by-product of metabolism changes the biochemical structure of a given lake. Nevertheless, the results may be the same: a change in the selective pressure experienced by organisms. The question is: how does this idea relate to the commensurability of ecological fitness? Does niche construction lead to incommensurability of fitness? And if so, how?

I think that niche construction, assuming it proceeds homogenously-i.e., when it changes the environment of all members of a given population in the same way-poses no challenge to the commensurability of ecological fitness. In this case, all organisms still have to solve the same design problems; it's just that the problems themselves have become somewhat different. However, I think that niche construction, when it proceeds heterogeneously-i.e., when it changes the environment of some members of a population, but not of all-poses a problem. In this case, it exposes some members of the population in question to different factors than others; as a result, the members thus exposed have to solve a different set of design problems than the others. Thus, the ecological fitness of the former becomes incommensurable with that of the latter. The evolution of heritable symbiosis offers an extreme example: the environment of a microorganism is changed through the action of a host that engulfs but does not digest it; rather, the host 'allows' the microorganism to live inside its body. As a result, the microorganism and its free-living counterparts are exposed to different obstacles, so that their fitness becomes incommensurable. Therefore, it may be said that the cause of the incommensurability of the ecological fitness of heritable symbionts and that of their free-living counterparts is niche construction which proceeds heterogeneously. This kind of niche construction tears a microorganism away from its old environment and places it inside a host, thus exposing it to environmental obstacles different than those faced by the members of its old population. This process is followed by the evolution of phenotypes, rendering any comparison of ecological fitness even less justified.

\section{Acknowledgments}

I would like to thank Javier Suárez, Pierrick Bourrat, Peter Godfrey-Smith, Derek Skillings, Thomas Pradeu, Jacob Stegenga, Gregor Greslehner, Maël Lemoine, Jean-François Moreau, Vanja Sisirak, Elena Rondeau, Wiebke Bretting, Lucie Laplane, and Nicolas Larmonier for the fruitful discussions and/or comments which greatly contributed to improving the final version of the paper. I declare no conflict of 
interest. This paper was financed by a National Science Centre Grant Opus 2017/27/B/HS1/00290 to A.S. Adrian Stencel's work is supported by the Foundation for Polish Science (FNP).

\section{Literature cited}

Ariew, A. and R. Lewontin. 2004. "The Confusions of Fitness." The British Journal for the Philosophy of Science 55: 347-363.

Bourrat, P. 2015. "Levels of Selection Are Artefacts of Different Temporal Fitness Measures." Ratio 28: 40--50.

Dyall, S. D., N. T. Brown, and P. Johnson. 2004. "Ancient Invasions: From Endosymbionts to Organelles." Science 304: 253-257.

Garcia, J. and N. Gerardo. 2014. "The Symbiont Side of Symbiosis: Do Microbes Really Benefit?" Frontiers in Microbiology 5: 1-6.

Godfrey-Smith P. 2014. Philosophy of Biology. Princeton: Princeton University Press.

Maynard-Smith, J., and E. Szathmary. 1995. Major Transitions in Evolution. Oxford: Oxford University Press.

Kawecki, T. J., R. E. Lenski, D. Ebert, B. Hollis, I. Olivieri, and M. C. Whitlock. 2012. "Experimental Evolution." Trends in Ecology and Evolution 27: 547-560.

Matthen, M., and A. Ariew. 2002. "Two Ways of Thinking About Fitness and Natural Selection." Journal of Philosophy 99: 55-83.

Kikuchi, Y., T. Hosokawa, and T. Fukatsu. 2007. "Insect-Microbe Mutualism Without Vertical Transmission: A Stinkbug Acquires a Beneficial Gut Symbiont From the Environment Every Generation." Applied and Environmental Microbiology 73: 4308-4316.

Laland, K., B. Matthews, and M. Feldman. 2016. "An Introduction to Niche Construction Theory." Evolutionary Ecology 30: 191-202.

Lowe, C., E. Minter, D. Cameron, and M. Brockhurst. 2016. "Shining a Light on Exploitative Host Control in a Photosynthetic Endosymbiosis." Current Biology 26: 207-211.

Moran, N., J. McCutheon, and A. Nakabachi. 2008. "Genomics and Evolution of Heritable Bacterial Symbionts.” Annual Review of Genetics 42: 165-190.

Mushegian, A., and D. Ebert. 2016. "Rethinking 'Mutualism' in Diverse Host-Symbiont Communities." BioEssays 38: 100-108

Odling-Smee, J. and K. Laland. 2011. "Ecological Inheritance and Cultural Inheritance: What Are They and How Do They Differ?" Biological Theory 6: 220-230.

Taylor, P. and R. Lewontin. 2017. “The Genotype/Phenotype Distinction.” In The Stanford Encyclopedia of Philosophy, Summer 2017 Edition, edited by Edward N. Zalta. https://plato.stanford.edu/ archives/sum2017/entries/genotype-phenotype/.

Templeton, A. 1989. “The Meaning of Species and Speciation: A Genetic Perspective.” In Speciation and Its Consequences, edited by D. Endler and J. Otte, 3-27. Sunderland: Sinauer Associates.

Triviño, V. and L. Nuño de la Rosa. 2016. "A Causal Dispositional Account of Fitness." History and Philosophy of the Life Sciences 38: 6.

Rosenberg, A. and F. Bouchard. 2015. "Fitness." In The Stanford Encyclopedia of Philosophy, Fall 2015 Edition, edited by Edward N. Zalta. https://plato.stanford.edu/archives/fall2015/entries/fitness/.

Stencel, A. 2016. The Relativity of Darwinian Populations and the Ecology of Endosymbiosis. Biology and Philosophy 31:619-637. 
Stencel, A. and Wloch-Salamon, D. 2018. Some Theoretical Insights Into Hologenome Theory of Evolution and the Role of Microbes in Speciation. Theory in Biosciences 137: 197-206.

Skillings, D. J. 2016. "Holobionts and the Ecology of Organisms-Multi-Species Communities or Integrated Individuals?" Biology and Philosophy 31: 875-292.

Suárez. J. 2016. "Bacterial Species Pluralism in the Light of Medicine and Endosymbiosis." Theoria 31(1): 91-205.

Suárez, J. 2018. "The Importance of Symbiosis in Philosophy of Biology: An Analysis of the Current Debate on Biological Individuality and Its Historical Roots." Symbiosis 76: 77-26.

Zimorski, V., C. Ku, W. F. Martin, and S. B. Gould. 2014. "Endosymbiotic Theory for Organelles Origin." Current Opinion in Microbiology 22: 33-28.

(C) 2022 Author(s)

This is an open-access article distributed under the terms of the Creative Commons Attribution 4.0 International license, which permits anyone to download, copy, distribute, display, or adapt the text without asking for permission, provided that the creator(s) are given full credit.

ISSN 2475-3025 